\title{
LES Investigation of Flow Field Sensitivity in a Gas Turbine Model Combustor
}

\author{
Yee Chee See* \\ Department of Aerospace Engineering, University of Michigan, Ann Arbor, MI 48109 \\ and Matthias Ihme ${ }^{\dagger}$ \\ Department of Mechanical Engineering, Stanford University, Stanford, CA 94305
}

\begin{abstract}
Large eddy simulation (LES) is a computational method that has the potential to enable the prediction of turbulent reacting flows in gas turbine combustors. However, flows in these complex combustor environments represent modeling challenges. Specifically, the vortex breakdown dynamics of swirling flows exhibit sensitivities to upstream and downstream conditions. Compounded by this is the added complexity of modern combustors, feature several geometrically-complex swirl generators. In the present work, a mesh sensitivity study is performed by considering a dual swirl gas turbine model combustor, which is studied at non-reacting flows conditions. By utilizing pure hexahedral meshes in the LES calculations, we are able to obtain results that show both grid convergence and agreement with experimental measurements. However, we also find that LES predictions of the flow field in this combustor can be highly dependent on the mesh type utilized for the simulations. Mesh refinement in crucial regions of the combustor was found to be insufficient to improve the simulation accuracy, and may - under certain circumstances - even worsen the modeling results. A parametric investigation of the mass flow rate split between the two swirlers only leads to the identification of a partial cause, which could be attributed to the presence of a subcritical bifurcation in the flow-field behavior.
\end{abstract}

\section{Introduction}

The advancement of gas-turbine (GT) engine technologies is primarily driven by the demand for higher power-densities, improved fuel-efficiencies and reduced environmental impact. Over recent years, most modern gas turbine developments have converged to a design solution that utilizes swirling flows in order to enhance mixing, increase flame-stability, and improve fuel efficiency. Furthermore, Gupta et al. ${ }^{1}$ emphasized that the advantages of swirl-stabilized combustion can further be increased by employing multiple co-annular streams of swirling flows. Thus, most modern engines exhibit complex designs that often contain more than one swirl generator.

However, the characteristics of swirling flows in combustors can be very sensitive to changes in geometry or inflow conditions. As example, minor modifications in the swirler, plenum, or injector arrangement can alter the flow field significantly. In an investigation on a combustor with movable block swirler and quarl, Vanoverberghe et al. ${ }^{2}$ categorized different patterns of flame stabilization over a range of swirl parameters. By manipulating the degree of swirl of the inflow in a specific manner, they observed a hysteresis in the flame pattern. Depending on the history of how the

${ }^{*}$ Research Assistant, AIAA member

${ }^{\dagger}$ Assistant Professor, AIAA member 
swirl parameter is changed, three different flame pattern are possible at the same swirl setting. In addition, they also observed an uncommon flame pattern at low to moderate swirl setting. This flame is attached to the burner face and wall and is attributed to the Coanda effect at the burner wall.

To extend the study of Vanoverberghe et al., ${ }^{2}$ Vanierschot and van den Bulck ${ }^{3}$ performed an isothermal swirling jet experiment using the same movable swirler. Although the present study does not consider heat release or confinement, a very similar hysteresis behavior was observed. Furthermore, the Coanda jet can be triggered by lowering the swirl strength from a high swirl configuration. This finding suggests that the Coanda jet is hydrodynamic in nature and can exist independent of combustion. In LES of a gas-turbine model combustor (GTMC), See and Ihme ${ }^{4}$ observed a similar attached flow-structure, which exhibited sensitivity to the sub-grid scale (SGS) model, and a similar flow-field structure was also obtained for the reacting cases using the same SGS model.

The objective of this work is to complement previous studies by characterizing the influence of computational mesh-topology on the predicted flow field of the GTMC. Specifically, meshes generated using different meshing strategies are considered here. Since the GTMC features two co-swirling streams of air, the sensitivity of the predicted flow field to the mass flow rate ratio between inner and outer swirler is investigated in this work. The LES methodology is summarized in the next section, which is then followed by a discussion of the experimental configuration and computational meshes in Sec. III. The results of the LES simulations for different meshes are presented in Sec. IV and the paper finishes with conclusions.

\section{Methodology}

The principle behind the LES methodology is to resolve large scale turbulent fluctuations while modeling the effects of the smaller computationally-unresolved scales. To achieve this, a spatial low pass filter is applied to the flow field quantities. The filtered value of a scalar $\psi$ is computed as:

$$
\widetilde{\psi}(t, \boldsymbol{x})=\frac{1}{\bar{\rho}} \int \rho(t, \boldsymbol{x}) \psi(t, \boldsymbol{x}) \mathcal{G}(t, \boldsymbol{x}, \boldsymbol{y} ; \Delta) d \boldsymbol{y},
$$

where $\Delta$ is the LES filter width and $\mathcal{G}$ is the low-pass filter. Applying this filtering procedure to the conservation equations, here written in a low-Mach number formulation, yields:

$$
\begin{aligned}
\widetilde{D_{t}} \bar{\rho} & =-\bar{\rho} \nabla \cdot \widetilde{\mathbf{u}} \\
\bar{\rho} \widetilde{D_{t}} \widetilde{\mathbf{u}} & =-\nabla \bar{p}+\nabla \cdot \underline{\underline{\tau}}-\nabla \cdot \underline{\underline{\tau}}^{\mathrm{res}},
\end{aligned}
$$

where $\mathbf{u}$ is the velocity vector, $\rho$ is the density, $p$ is the pressure, $D_{t} \equiv \partial_{t}+\mathbf{u} \cdot \nabla$ is the substantial derivative, $\underline{\underline{\tau}}$ is the viscous shear stress tensor, and the superscript "res" refers to the residual stresses. These residual stresses require modeling. In the following, $\underline{\tau}^{\text {res }}$ is evaluated using the eddy-viscosity model, i.e.,

$$
\underline{\underline{\tau}}^{\mathrm{res}}=\bar{\rho} \widetilde{\mathbf{u}} \widetilde{\mathbf{u}}-\bar{\rho} \widetilde{\mathbf{u u}}=2 \bar{\rho} \nu_{t} \underline{\underline{\widetilde{S}}}
$$

where $\underline{\underline{S}}$ is the Favre-filtered strain rate and $\nu_{t}$ is the turbulent viscosity.

In the present study, we utilize the Vreman model ${ }^{5}$ to estimate the residual turbulent stresses. In this model, the turbulent viscosity is computed as:

$$
\nu_{t}=C_{v}\left(\frac{\beta_{11} \beta_{22}-\beta_{12}^{2}+\beta_{11} \beta_{33}-\beta_{13}^{2}+\beta_{22} \beta_{33}-\beta_{23}^{2}}{\frac{\partial \widetilde{u}_{k}}{\partial x_{l}} \frac{\partial \widetilde{u}_{k}}{\partial x_{l}}}\right)^{1 / 2}
$$


where

$$
\beta_{i j}=\Delta^{2} \frac{\partial \widetilde{u}_{k}}{\partial x_{i}} \frac{\partial \widetilde{u}_{k}}{\partial x_{j}}
$$

The constant model coefficient $C_{v}$ is set to 0.07 in this study. This turbulent model is developed to yield vanishing turbulent viscosity for thirteen types of laminar flow configurations. One of the flow configuration is flow near a wall and this property may be needed for the accurate prediction of separated flows in the combustion chamber of the GTMC.

To obtain numerical solutions of the governing equations, the variable density solver VIDA was employed in this study. This LES solver utilizes a second-order skew-symmetric spatial discretization $^{6}$ on unstructured meshes. The spatial scheme has low numerical dissipation and hence is suitable for application in LES with explicit SGS model. For the temporal discretization, the low Mach number approximation is assumed so that the pressure Poisson equation is solved at each time-step. ${ }^{7}$ The low Mach number approximation decouples the acoustic propagation from the flow dynamics and thus reduces the stiffness of the equations solved.

Boundary conditions at the plenum inlet and at the fuel injector are specified with mass flow rates discussed in Sec. III.A. Furthermore, no turbulence is imposed at the inlets and constant inflow profiles are prescribed. Convective outflow boundary conditions are used at the combustor exit. No wall-model is used in this study and the no-slip condition for velocity is simply imposed at the combustor walls.

\section{Experiment Configuration and Computational Meshes}

\section{III.A. Experimental Setup}

In this work, we considered the gas-turbine model combustor (GTMC) that was experimentally investigated by Meier and coworkers. ${ }^{8,9}$ A schematic of the burner is illustrated in Fig. 1. The injector consists of a central air nozzle, an annular fuel nozzle, and a co-annular air nozzle. Both air nozzles supply swirling air at ambient temperature from a common plenum. The inner air nozzle has a diameter of $15 \mathrm{~mm}$; the annular nozzle has an inner diameter of $17 \mathrm{~mm}$ and an outer diameter of $25 \mathrm{~mm}$. Nonswirling fuel is provided through three exterior ports that are fed through the annular nozzle. The exit plane of the central air nozzle and fuel nozzle lies $4.5 \mathrm{~mm}$ below the exit plane of the outer air annulus. The combustion chamber has a square cross section of $85 \mathrm{~mm}$ in width and $110 \mathrm{~mm}$ in height. The exit of the combustion chamber is an exhaust tube with a diameter of $40 \mathrm{~mm}$ and a height of $50 \mathrm{~mm}$.

Instead of fuel, air is supplied through the fuel injector ports in the non-reacting case. The mass flow rates through the central air nozzle and the annular fuel nozzle are $19.74 \mathrm{~g} / \mathrm{s}$ and 1.256 $\mathrm{g} / \mathrm{s}$, respectively. ${ }^{10}$ The inlet temperature of the mixture is 300

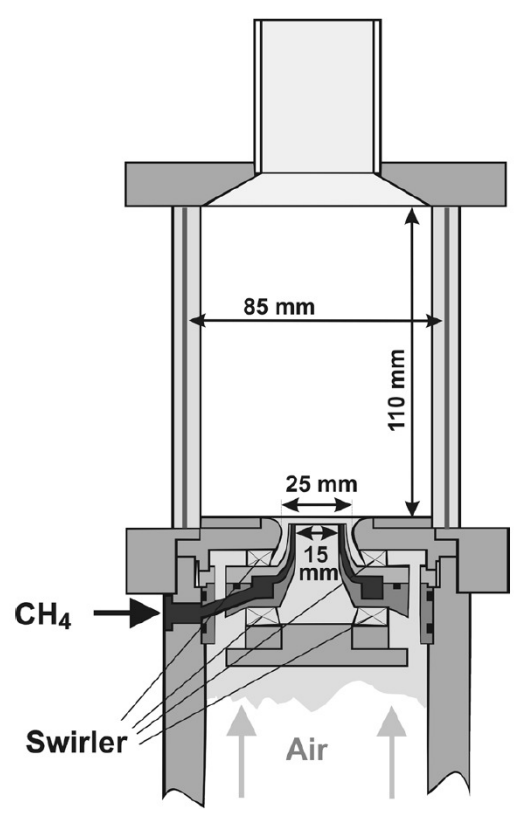

Figure 1: Schematic of gas turbine model combustor. ${ }^{8,9}$

$\mathrm{K}$, and the burner is operated at a pressure of 1 bar. At this condition, the flow field inside the combustion chamber resembles a "type B" flow pattern, which was categorized by Beer \& Chigier. ${ }^{11}$ This flow field is characterized by an internal recirculation zone (IRZ) that is established at the axis due to the vortex breakdown. In addition, an outer recirculation zone (ORZ) is also present in this configuration as the injector stream is detached from the burner face. 


\section{III.B. Computational Meshes}

Two different types of unstructured meshes are considered in this study to assess the sensitivity of the LES predictions to the underlying discretization. The first mesh is generated using a multiblock meshing strategy. All elements in this mesh are hexahedral. However, due to the geometric complexity, the generation of this block topology for the entire burner is a time-consuming process. Moreover, the element distributions in a multi-block mesh, without local refinement, can be constrained by the block topology. In this study, a skeletal multi-block mesh is first generated and locally refined in regions of importance to yield meshes I1, I2, and I3.

The second mesh generation approach considered is to use a combination of multi-block meshes and fully unstructured tetrahedral meshes. This hybrid mesh generation method allows for a significantly faster generation of pure tetrahedral meshes for complex flow passages while retaining hexahedral meshes in regions of the computational domain where the geometry is simpler. To this end, the hybrid mesh $\mathrm{H} 1$ is generated by combining a fully tetrahedral mesh of the swirler with multi-block meshes of the plenum and the combustion chamber. A refined version of the mesh H1 is also considered in this study, which is denoted as mesh H2. In this refined mesh, elements within and near the swirler are refined so that the maximum edge length of these elements does not exceed $0.6 \mathrm{~mm}$.

The element distribution among the three regions of the combustor are shown Tab. 1 for all the meshes considered in this work. For the fully hexahedral meshes, most of the elements are localized in the swirlers. However, the coarsest hybrid mesh has more elements in the combustion chamber than in the swirlers. The assessment of the mesh resolution in Sec. IV.C reveals that the swirler region is under-resolved for this hybrid mesh. Therefore, mesh $\mathrm{H} 2$ is constructed to increase the mesh resolution in the swirlers.

Although meshes I2 and $\mathrm{H} 2$ are of different mesh type, the element distribution for these two meshes is comparable. Therefore, a planar cut along the center-plane for $z=0$ is shown in Fig. 2 to highlight the difference between the two meshes. The pure tetrahedral mesh in the swirlers appears to be denser in this cut plane but this is an artifact of the cut plane intersecting with more tetrahedral elements. During the mesh generation process for both mesh types, we have also ensured sufficient wall resolution at the swirler nozzle region where the flow can separate. This can be seen in Fig. 3 where planar cuts of the meshes show the stretching of the mesh to achieve sufficiently small wall spacing.

\begin{tabular}{ccccc}
\hline & \multicolumn{4}{c}{ Number of elements (in million) } \\
\cline { 2 - 5 } Mesh & Plenum & Swirlers & Combustion chamber & Total \\
\hline \hline I1 & 0.5 & 6 & 1.5 & 8 \\
I2 & 2 & 10 & 5 & 17 \\
I3 & 2 & 20 & 21 & 43 \\
H1 & 0.5 & 2 & 4.5 & 7 \\
H2 & 2 & 12 & 6 & 20 \\
T1 & 0 & 8 & 7 & 15 \\
\hline
\end{tabular}

Table 1: Element distribution of pure hexahedral meshes (I1, I2, I3) and hybrid meshes (H1, H2). Element distribution of mesh $\mathrm{T} 1$ for the truncated burner geometry is also shown.

To study the flow field sensitivity to the air flow distribution between inner and outer swirler, a truncated domain of the GTMC is considered here. In this smaller computational domain, the two swirlers no longer share a common plenum so that the mass flow rates through each swirler is individually prescribed. The geometry of the truncated combustor assembly is illustrated in Fig. 4a and the simulation domain now begins from the swirl vanes. The mesh for this simulation domain 


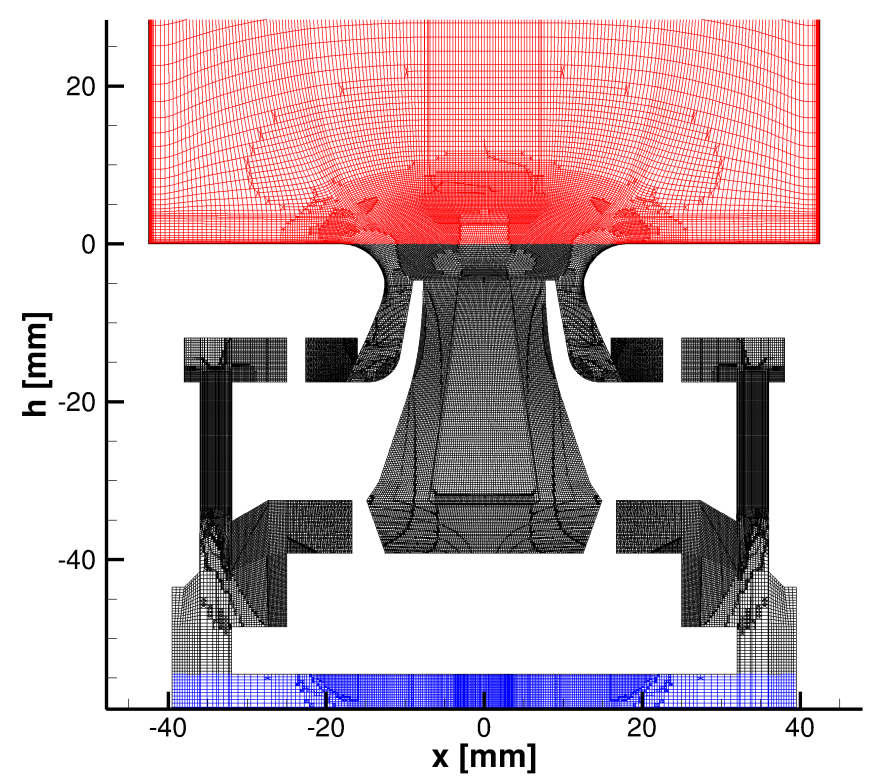

(a) Mesh I2.

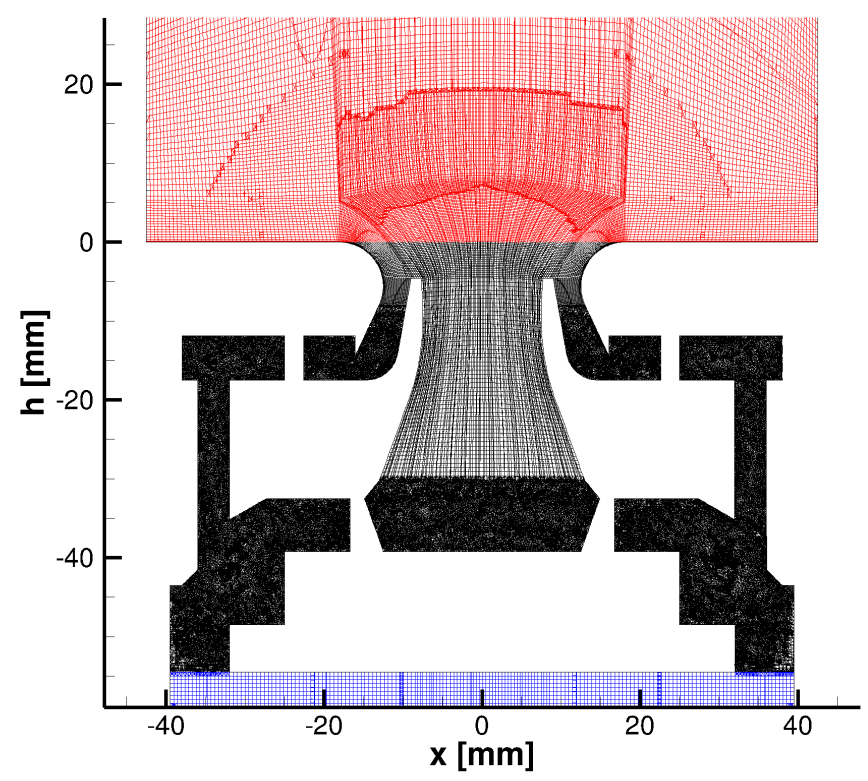

(b) Mesh H2.

Figure 2: Illustration of meshes I2 and $\mathrm{H} 2$ in the swirler section of the combustor. $h$ denotes axial distance from the burner face.

is shown in Fig. 4b. The mesh for the truncated burner is largely based on the mesh H2, and the computational mesh inside the combustion chamber is refined to resolve the shear-layer region. 


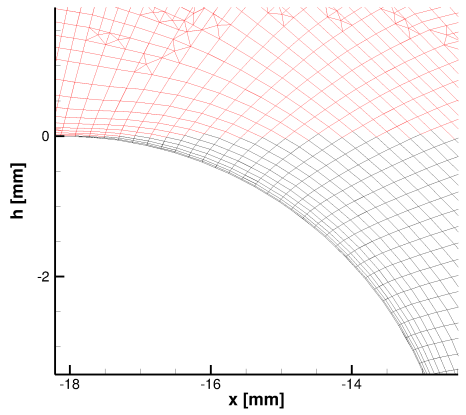

(a) Mesh I2.

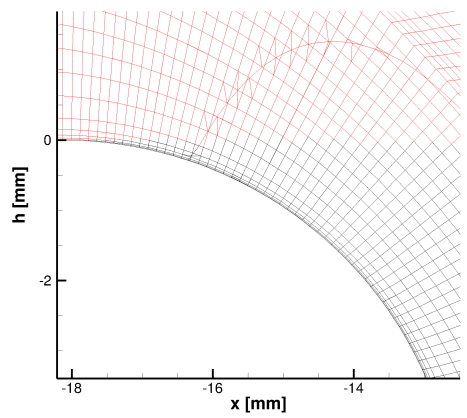

(b) Mesh $\mathrm{H} 2$.

Figure 3: Wall resolution of meshes I2 and $\mathrm{H} 2$ at the outer injector.

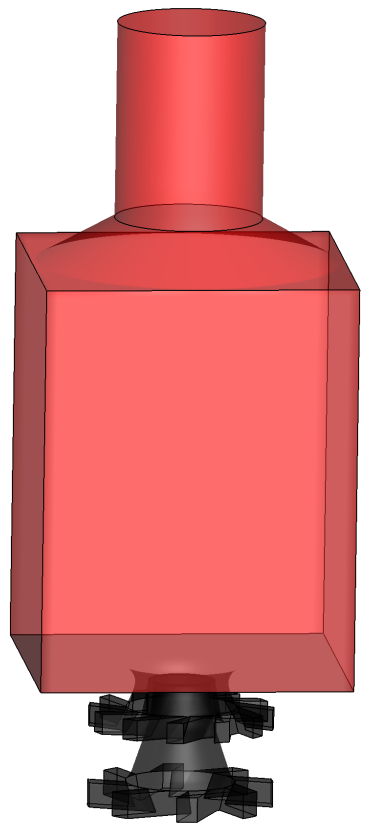

(a) Computational domain of the truncated burner.

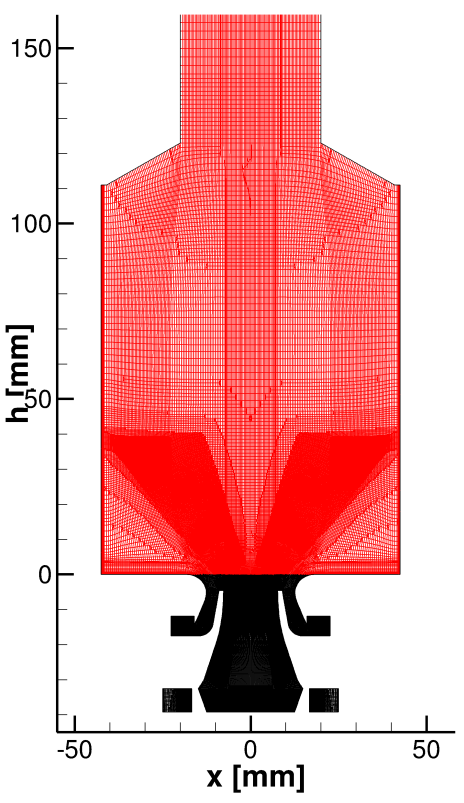

(b) Mesh T1.

Figure 4: Truncated computational domain, showing (a) 3D-rendering and (b) computational mesh T1.

\section{Results}

\section{IV.A. Flow Field Structure}

A comparison of the mean axial velocity fields along the burner centerplane $(z=0)$, obtained from the three meshes I1, I2, and I3, are shown in Fig. 5. Overall, these computations are able to reproduce the key flow field features observed in the experiment. Specifically, the injector stream from the swirlers is initially separated but re-attaches to the wall at a location further downstream. As a result of this flow separation, an ORZ is formed in the lower corner of the combustion chamber. The vortex breakdown phenomena, induced by the sudden expansion of the outer swirler nozzle wall, leads to the formation of an IRZ. This IRZ can be seen as a Y-shaped region of negative axial velocity in Fig. 5. 
LES predictions for mean axial velocity fields on meshes $\mathrm{H} 1$ and $\mathrm{H} 2$ are shown in Fig. 6. Similar to the LES results on the hexahedral mesh, the mean velocity field on mesh $\mathrm{H} 1$ also shows reasonable agreement with the experimental data. However, the simulation on mesh H1 predicts an ORZ that is slightly larger than that obtained for the hexahedral meshes. This may be attributed to larger mass flow rates through the inner swirler, which is further discussed in Sec. IV.D. However, the simulation results on mesh $\mathrm{H} 2$ is considerably different from LES solutions on the other meshes that are considered here. Specifically, the mean axial velocity fields on mesh H2 shows that the injector stream is always attached to the wall, resulting in the formation of a Coanda jet. As a result, the ORZ is no longer present in the combustion chamber. The reason for this discrepancy is further explored in Sec. IV.D.

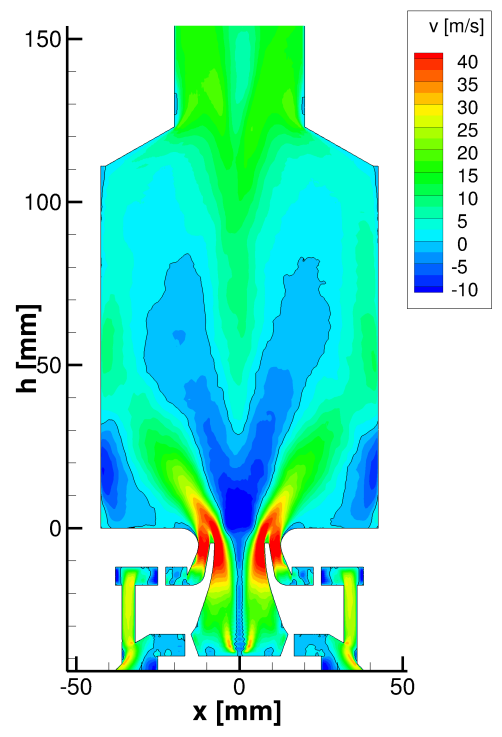

(a) Mesh I1.

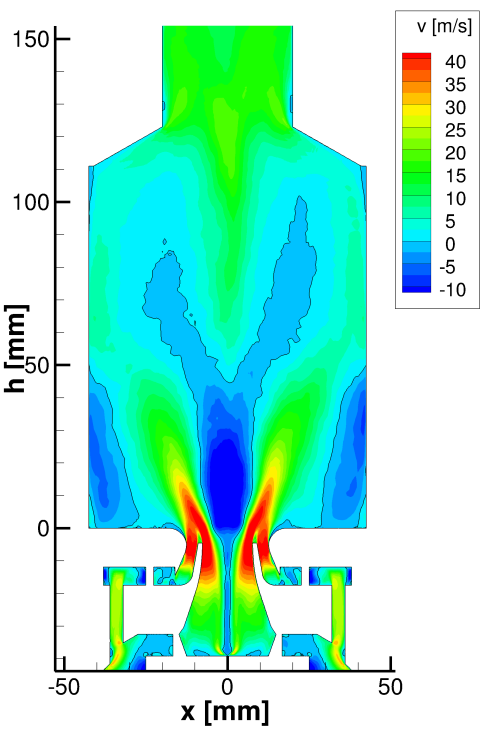

(b) Mesh I2.

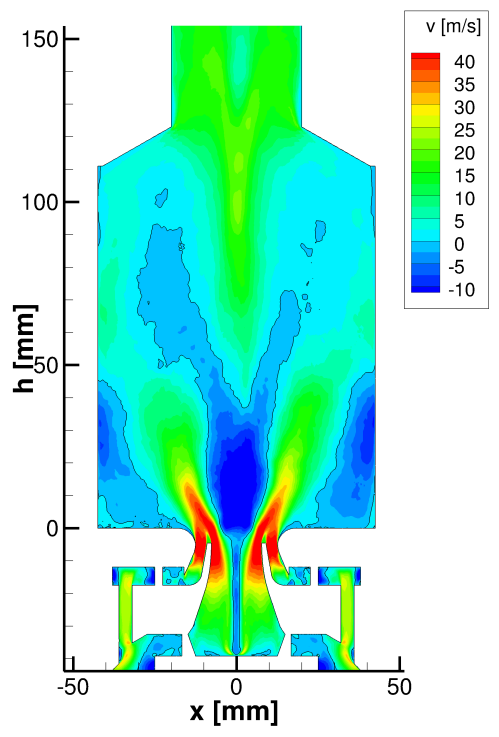

(c) Mesh I3.

Figure 5: Mean axial velocity on (a) mesh I1, (b) mesh I2, and (c) and mesh I3. The iso-line of zero axial velocity is shown as an indicator of the recirculation zone.

\section{IV.B. Comparison of Velocity Statistics}

The statistics for each simulation are collected for at least one flow-through-time to ensure sufficient convergence of the first statistical moments on measurement locations lower than $h=20 \mathrm{~mm}$. A comparison of the mean-flow profiles from the simulations using different mesh-representations and experiments are shown in Fig. 7. Overall, the LES results on meshes I2 and I3 are in good agreement with experimental results. However, the LES calculation on the mesh I1 yields an axial velocity profile that is shifted slightly outward in the radial direction at $h=20 \mathrm{~mm}$. Nevertheless, the timeaveraged LES predictions tend to approach the experimental measurements with increasing mesh resolution. At the last axial measurement state of $h=90 \mathrm{~mm}$, some discrepancies can be seen in the mean velocity profiles which can be attributed to the incomplete statistical convergence. Excluding this location, this comparison generally shows that the mean results are mostly grid-converged on the refined mesh I3.

The mean velocity profiles predicted with mesh $\mathrm{H} 1$ also shows reasonable agreement with measurements. The velocity maxima at $h=5 \mathrm{~mm}$ and $10 \mathrm{~mm}$ are overpredicted, and the simulation results indicate a stronger reverse flow at the centerline and lower radial spreading of the injector stream at these locations. These features are characteristics of a larger ORZ as shown in Fig. 6a. 


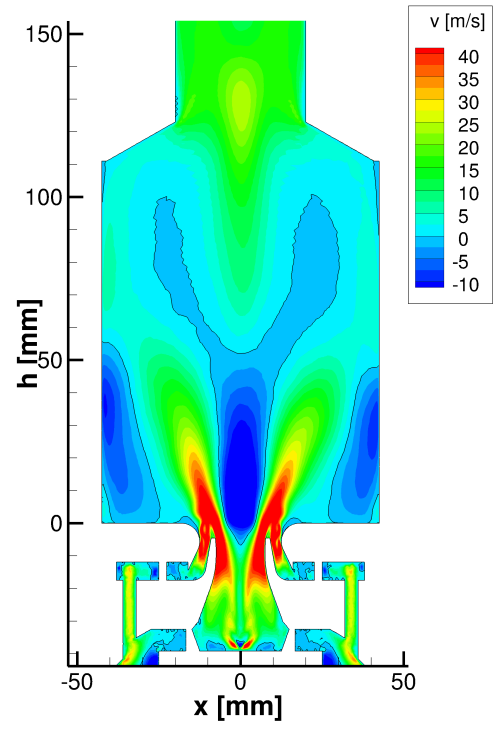

(a) Mesh H1.

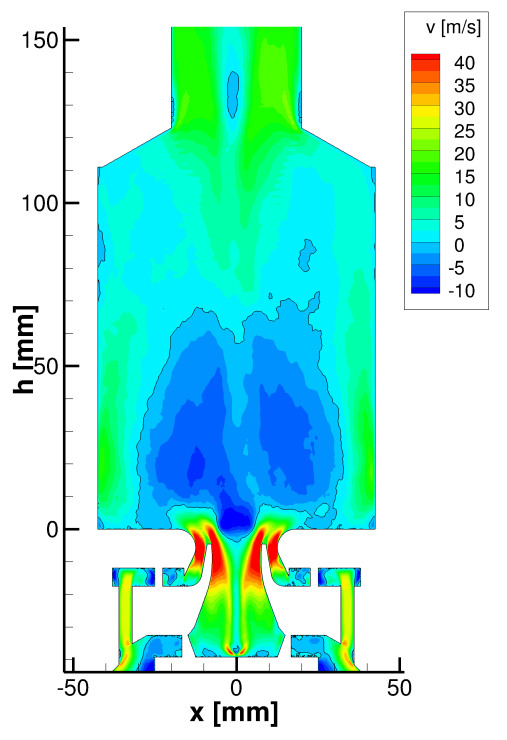

(b) Mesh H2.

Figure 6: Mean axial velocity on (a) mesh $\mathrm{H} 1$ and (b) mesh $\mathrm{H} 2$. The iso-line of zero axial velocity is shown as an indicator of the recirculation zone.

The Coanda jet predicted with mesh $\mathrm{H} 2$ results in mean velocity profiles that do not agree with experimental data. Despite this, the mean profile at the downstream location of $h=90 \mathrm{~mm}$ are well-captured with this mesh, indicating that the flow field is mostly determined by the exhaust nozzle geometry at this point.

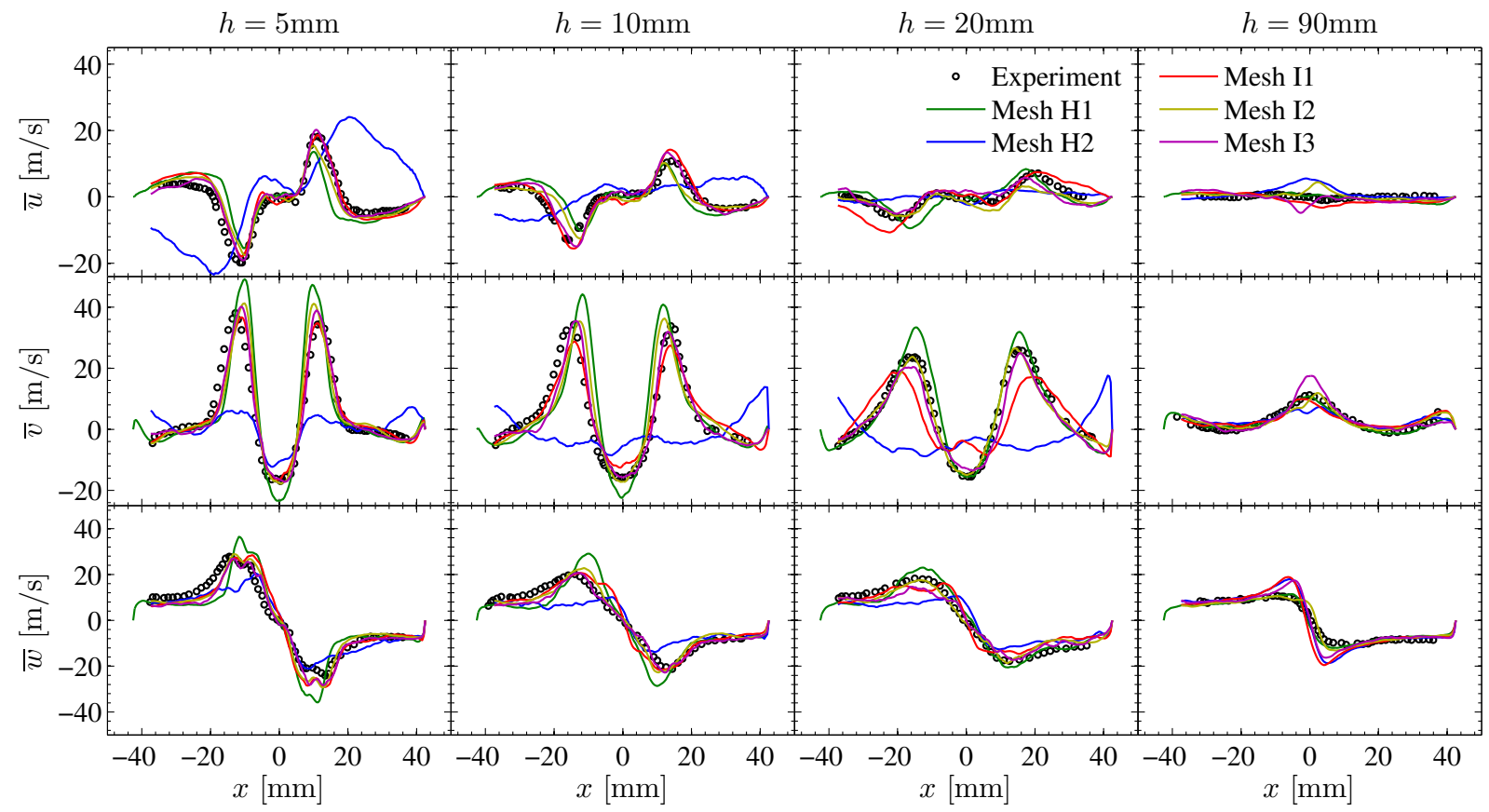

Figure 7: Comparison of mean velocity for simulations with LDV measurements at the cut-plane of $z=0$. $v$ is axial velocity while $u, w$ represent the radial velocity and tangential velocity in the cut-plane of $z=0$, respectively. 


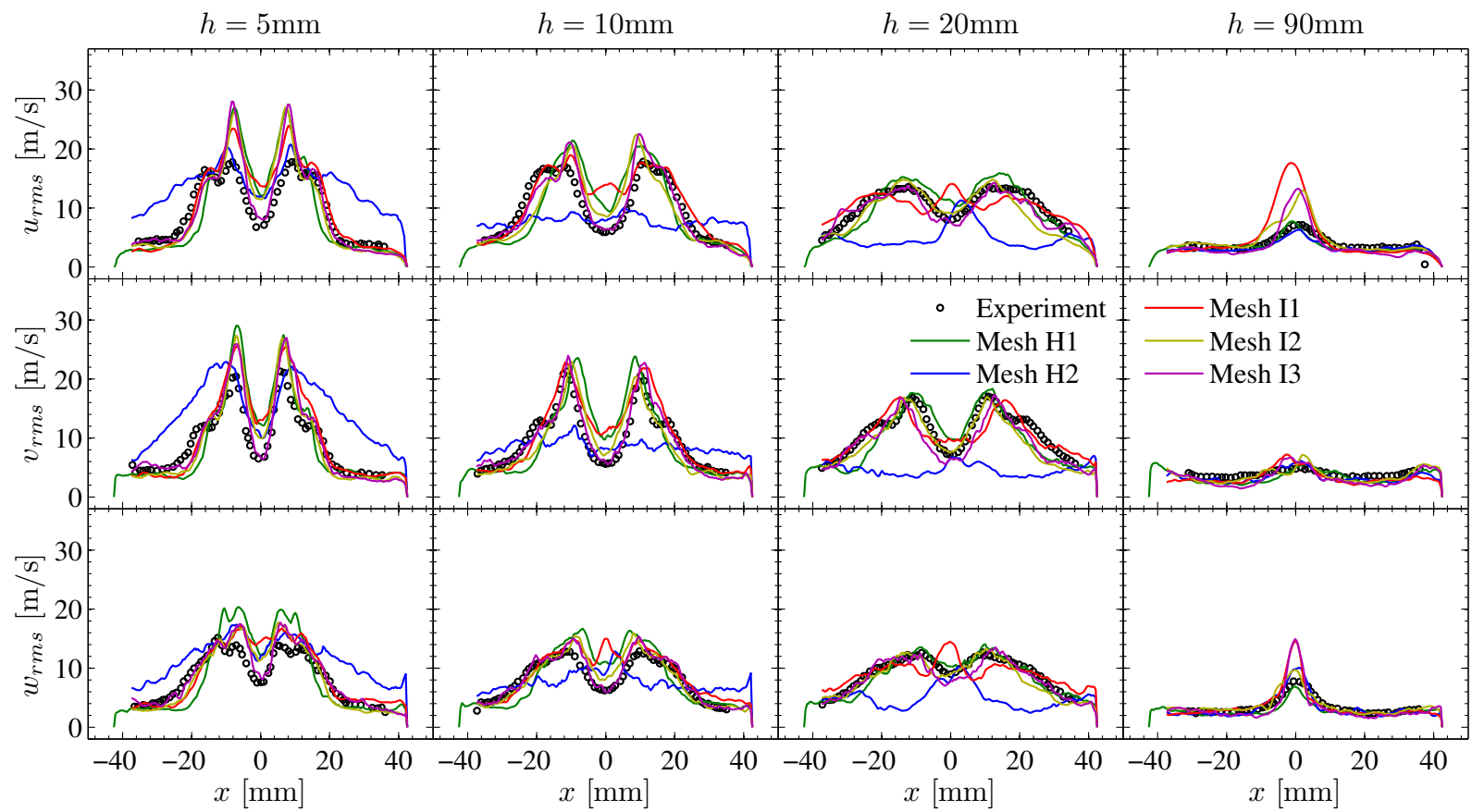

Figure 8: Comparison of resolved root mean squared (rms) of velocity for simulations with LDV measurements at the cut-plane of $z=0 . v$ is axial velocity while $u, w$ represent the radial velocity and tangential velocity in the cut-plane of $z=0$, respectively.

Figure 8 shows the resolved root mean squared (rms) velocity statistics from the LES calculations in comparison with experimental measurements. At measurement locations $h=10 \mathrm{~mm}$ and $h=20 \mathrm{~mm}$, the velocity fluctuations obtained from LES calculations on meshes I2 and I3 show excellent agreement with experimental measurements. The LES results on mesh I1 over-predict the fluctuations of velocity at the centerline. At $h=5 \mathrm{~mm}$, the peaks of velocity fluctuations are slightly over-predicted by the LES calculations. The predictions of the rms velocity at the centerline show improvements with grid refinement but the velocity fluctuations around $x= \pm 15$ $\mathrm{mm}$ remain high even after grid refinement. This over-prediction of the velocity fluctuations may be attributed to the lower turbulent eddy viscosity computed with the Vreman turbulence model.

The LES calculation on mesh H1 also shows similar agreement with measurements. The overprediction of velocity fluctuations is also observed here as the Vreman SGS model is utilized in this simulation. Due to the different flow topology predicted on mesh H2, the rms velocity profiles do not show agreement with experimental data except at $h=90 \mathrm{~mm}$. At $h=5 \mathrm{~mm}$, the rms velocity profiles for mesh $\mathrm{H} 2$ are close to the experimental values for $|x|<=20 \mathrm{~mm}$ but are much higher for $|x|>20 \mathrm{~mm}$. Since the injector stream does not extend to $h=10 \mathrm{~mm}$ and $20 \mathrm{~mm}$ in the simulation on mesh $\mathrm{H} 2$, the fluctuations induced by shear are absent, resulting in lower velocity fluctuations at these two locations.

\section{IV.C. LES Quality}

Pope $^{12}$ proposed a criterion to evaluate the quality of the LES calculations. This metric, $M(\boldsymbol{x}, t)$, is defined as:

$$
M=\frac{k^{\mathrm{res}}}{K+k^{\mathrm{res}}}
$$

where $K$ is the resolved turbulent kinetic energy and $k^{\text {res }}$ is the turbulent kinetic energy captured by the turbulence model. The value of $M$ is bounded between 0 and 1 , and $M=0$ corresponds to 
a fully resolved simulation (DNS) while $M=1$ corresponds to a simulation in which the turbulence is fully modeled, e.g. Reynolds-averaged Navier-Stokes (RANS) simulation.

The resolved turbulent kinetic energy can be obtained in LES by evaluating the temporal velocity statistics, but $k^{\text {res }}$ is usually approximated using models. From dimensional arguments, $k^{\text {res }}$ can be estimated as:

$$
k^{\mathrm{res}}=\left(\frac{\nu_{t}}{C_{k} \Delta}\right)^{2}
$$

The turbulent viscosity can be evaluated with the same sub-grid scale turbulence models mentioned in Sec. II, and $C_{k}$ is a model coefficient. Here, $C_{k}$ is set to 0.089 , which is close to a previously reported value. ${ }^{13}$

Pope's criterion for LES calculations on all meshes are computed with Eq. (6) and the planar fields of this quantity at $z=0$ are shown in Fig. 9. In addition, the iso-contour for the thresholdvalue of $M=0.2$ is shown in Fig. 9 to delineate the region in the combustor where the large scale turbulence is well-resolved and where it is not. This cutoff value is adopted as a metric to assess the quality of LES. ${ }^{14}$

Overall, the computed criterion for the LES calculations on the hexahedral meshes are mostly below 0.2 in the interior of the combustion chamber and swirlers. However, this metric also indicates that there is more unresolved turbulence fluctuation near the centerline of the inner swirler nozzle. This is due to the strong rotation of the flow in this area and the tendency of the Vreman model to over-estimate the turbulent viscosity in flows involving solid body rotation. ${ }^{15}$ Nevertheless, the grid refinement of this region in mesh I3 seems to reduce Pope's criterion below the recommended threshold.

Pope's criterion evaluated on mesh H1 shows that the swirler region is under-resolved. The modeled turbulent viscosity in this region is sufficiently high, suggesting that this LES computation behaves more like a RANS simulation in the swirlers. Although the flow field in the combustion chamber is well-predicted by this LES, this characteristic is undesirable for high fidelity simulations. Therefore, this hybrid mesh H1 is further refined to obtain the mesh H2. Pope's criterion computed on mesh $\mathrm{H} 2$ is more appropriate for a LES calculation. Nevertheless, $M$ is still high in the inner swirler nozzle as the turbulence model remain unchanged. If one is not informed about the experimental measurements, it might be tempting to conclude that the Coanda jet is the likely flow field but this is clearly not true. Therefore, the usage of Pope's criterion to determine the quality of an LES computation should be approached with caution as this metric is a local assessment of the simulation quality. As demonstrated here, the accuracy of the LES prediction of the more global flow field is not captured by this quality metric.

\section{IV.D. Swirl Number and Mass Flow Rate Split}

Statistical comparisons (shown in Sec. IV.B) are mostly restricted to the flow field inside the combustion chamber but the flow conditions upstream can be crucial in determining the flow dynamics inside the chamber. To this end, the split of air mass flow rate between the inner and outer swirler is characterized here. For this, a mass-flow split ratio, $\dot{m}_{r}$, is introduced:

$$
\dot{m}_{r}=\frac{\dot{m}_{\text {out }}}{\dot{m}_{\text {in }}}
$$

where $\dot{m}_{\text {in }}$ and $\dot{m}_{\text {out }}$ are the mass flow rates through the inner and outer swirler, respectively.

The swirl number is a characterization of the degree of swirl in a flow and is a crucial parameter in the investigation of swirling flows. When first introduced, the swirl-number was defined ss $^{16}$

$$
S_{C B}=\frac{G_{t g}}{G_{a x}}=\frac{\int \rho w u r d A}{R \int \rho\left(u^{2}+p\right) d A},
$$

10 of 15 


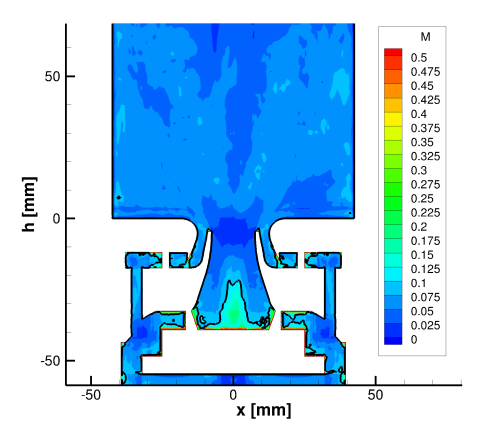

(a) Mesh I1.

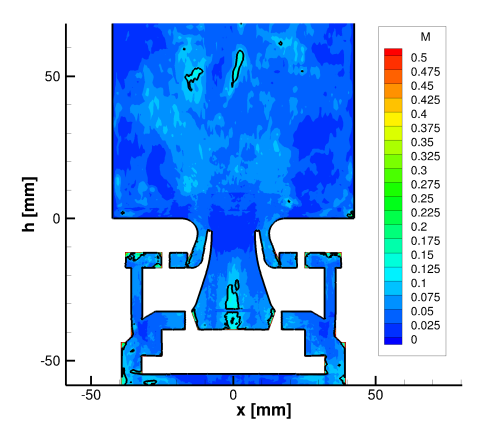

(b) Mesh I2.

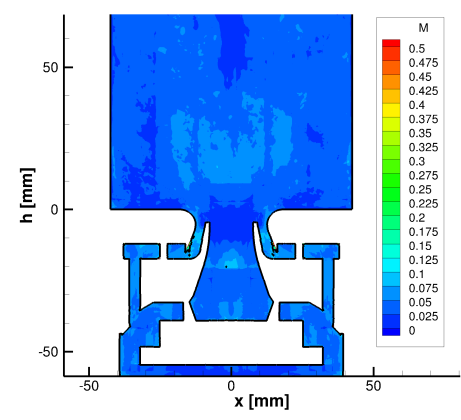

(c) Mesh I3.

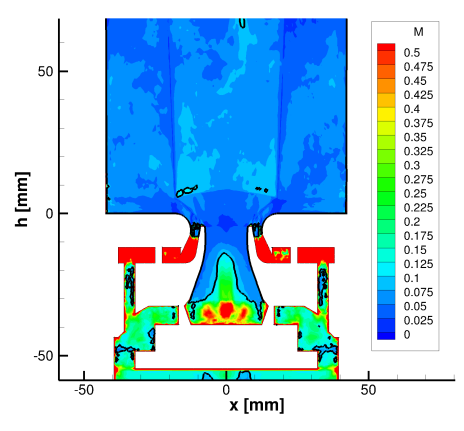

(d) Mesh H1.

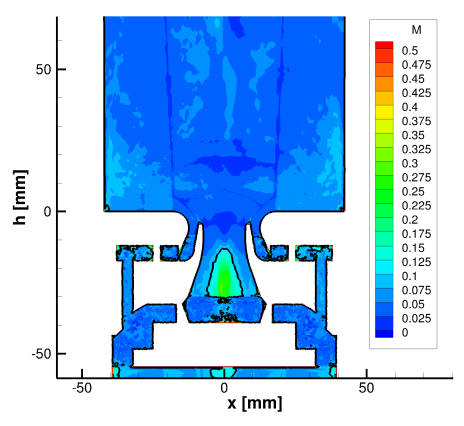

(e) Mesh H2.

Figure 9: Comparison of Pope's criterion $M$ for different meshes I1, I2, I3, H1 and H2, considered in this study. Black lines indicate the constant value of $M=0.2$.

where $G_{t g}$ is the tangential momentum flux, $G_{a x}$ is the axial momentum flux, $r$ is the radial position, $R$ is the outer radius, $w$ is the azimuthal velocity, $u$ is the axial velocity, and $p$ is the pressure. Due to the difficulty in obtaining static pressure measurements to calculate the axial momentum in the swirl generators, this ratio was simplified ${ }^{11}$ to the more commonly utilized expression of swirl number $S$, i.e.

$$
S=\frac{\int \rho w u r d A}{R \int \rho u^{2} d A} .
$$

The swirl number for each swirler has been evaluated individually at $h=-5.5 \mathrm{~mm}$. This location lies below the fuel injectors where the inner and outer swirler streams have yet to be merged. By computing the swirl numbers separately for each stream, the inner and outer swirl numbers can be obtained. The total swirl number is evaluated at $h=4.5 \mathrm{~mm}$ where the flow has merged into a single stream.

The swirl numbers and mass flow rate ratios from all simulations are summarized in Tab. 2. This table shows that the LES calculations on meshes I2 and I3 predict similar swirl numbers but $\dot{m}_{r}$ of the LES solution on mesh I2 is lower than that of mesh I3. Although the simulations on meshes I1 and I3 predict similar $\dot{m}_{r}$, the swirl numbers are higher for mesh I1. As the vortex breakdown dynamics can depend on the degree of swirl of a flow, ${ }^{17}$ the higher swirl number may explain the larger IRZ predicted by the simulation on mesh I1.

The mass flow rate ratio evaluated for LES results on mesh H1 is significantly lower than the other simulations. This may be attributed to the lack of mesh resolution in the swirlers as the higher turbulent viscosity in one of the swirler paths may impede the flow through it. As a result of the 


\begin{tabular}{ccccc}
\hline & & \multicolumn{3}{c}{ Swirl number } \\
\cline { 3 - 5 } Mesh & $\dot{m}_{r}$ & Inner & Outer & Total \\
\hline \hline I1 & 1.51 & 0.422 & 0.945 & 0.714 \\
I2 & 1.44 & 0.396 & 0.903 & 0.671 \\
I3 & 1.50 & 0.400 & 0.904 & 0.676 \\
H1 & 1.26 & 0.418 & 0.969 & 0.700 \\
H2 & 1.55 & 0.385 & 0.910 & 0.690 \\
\hline
\end{tabular}

Table 2: Swirl numbers and mass flow rate ratios for LES calculations on hexahedral meshes I1, I2, and I3, and hybrid meshes $\mathrm{H} 1$ and $\mathrm{H} 2$.

lower mesh resolution, the predicted swirl number is generally higher than that of the simulations on the finer meshes. Moreover, the lower $\dot{m}_{r}$ can also be a factor for the larger ORZ predicted by this simulation and this is further examined in Sec. IV.E

Although the flow field from mesh $\mathrm{H} 2$ exhibits a significantly different flow-structure, the mass flow rate ratio for this case does not deviate significantly from that of the fully hexahedral meshes. This suggests that the mass flow rate split between the two swirlers may not be the cause for the different flow field predictions. The comparison of the swirler numbers also does not reveal any trend that suggest these parameters are responsible for the Coanda jet.

\section{IV.E. Results of Truncated Domain Simulation}

In Sec. IV.D, the mass flow rate split between the two swirlers is identified as a possible factor in determining the characteristics of the recirculation zones in the combustor. To analyze this further, we consider LES computations of the truncated burner (shown in Fig. 4a), where the mass flow rates through each swirler can be independently varied. Five different mass flow rate ratios, summarized in Tab. 3, have been investigated in this study in order to elucidate the effects of this parameter on the flow field inside the combustion chamber.

The mean axial velocity field for the five simulations of different $\dot{m}_{r}$ are shown in Fig. 10. In the simulations for $\dot{m}_{r} \leq 1.4$, the injector stream separates from the outer swirler nozzle. With increasing shift in the mass flow rate towards the outer swirler, the separated flow re-attaches to the combustor chamber at locations closer to the bottom wall. For cases of $\dot{m}_{r} \geq 3.0$, the flow almost never separates from the wall, thus eliminating the ORZ.

To quantify the effect of mass flow split between the swirlers, swirl numbers are evaluated as described in Sec. IV.D and are shown in Tab. 3. With the exception of the limiting cases of $\dot{m}_{r}=0$ and $\dot{m}_{r}=\infty$, the swirl number for each swirler is relatively insensitive to the variation of mass flow rates. Since the geometry of the swirlers is fixed in this study, it is expected that the degree of swirl imparted by the swirlers remains unchanged. However, the total swirl number of the flow is increased when more air is flowing through the outer swirler. A comparison of the swirl numbers for each swirler reveals that the outer swirler seems to generate more swirl than the inner swirler. Hence, a mass flow rate split that favors the outer swirler can lead to a larger total swirl number.

Previous studies on swirling flows ${ }^{1,11}$ have shown that the increase in the swirl-strength can cause a flow transition from a separated swirling jet (Type B flow) into a Coanda jet (Type C flow). In the truncated geometry, the increase in the total swirl number is also correlated with the transition to an attached flow. Hence, this flow behavior elucidated through LES studies is consistent with the findings of previous work. ${ }^{11}$ In summary, the mass flow rate split can affect the flow field within the combustor chamber by changing the total swirl number of the flow.

It is also noteworthy to point out that the simulation of the truncated burner at $\dot{m}_{r}=1.4$ shares similar swirl numbers and mass-flow splits with the full combustor simulation on mesh I2. Despite 
this, the predicted mean axial velocity field in the truncated domain shows a ORZ that is smaller than that of the full combustor simulation. Clearly, a more comprehensive study is required to identify additional factors that can contribute to a different flow field in the combustion chamber.

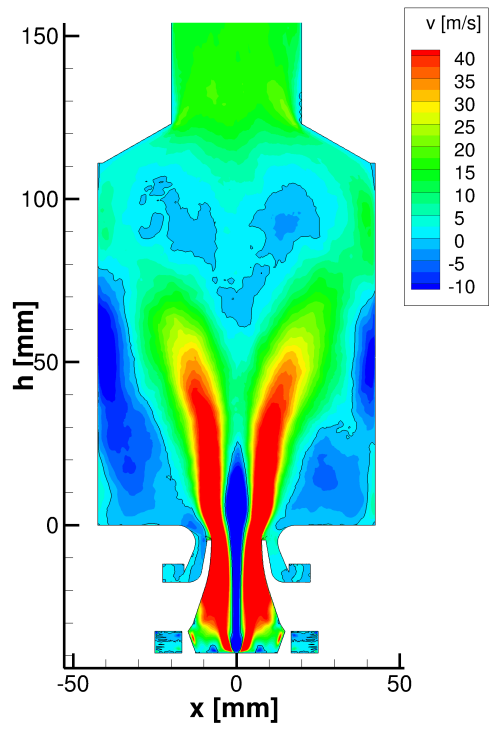

(a) $\dot{m}_{r}=0.0$.

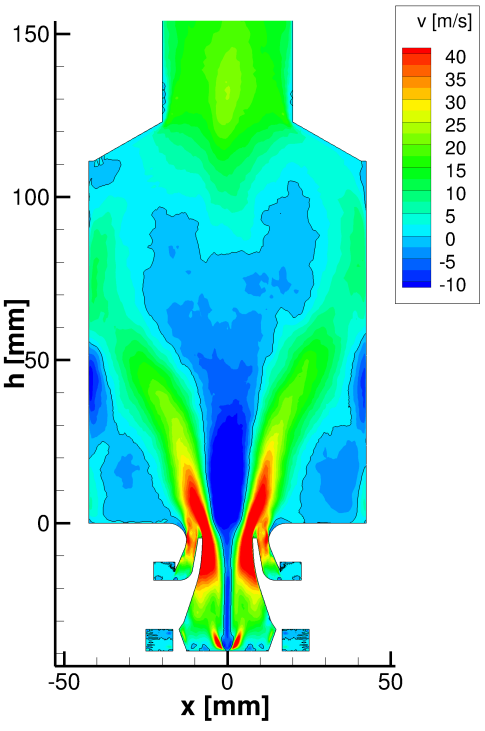

(b) $\dot{m}_{r}=1.0$.

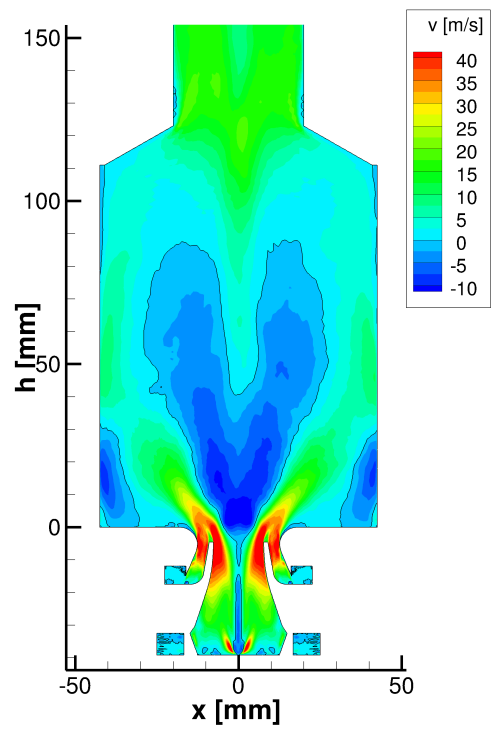

(c) $\dot{m}_{r}=1.4$.

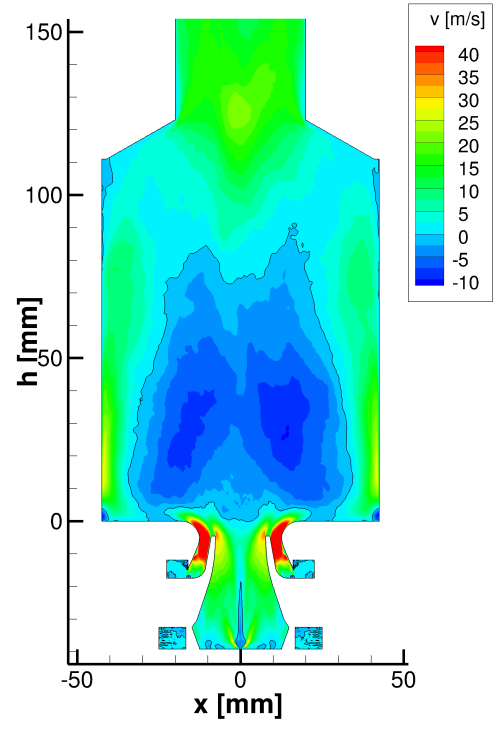

(d) $\dot{m}_{r}=3.0$.

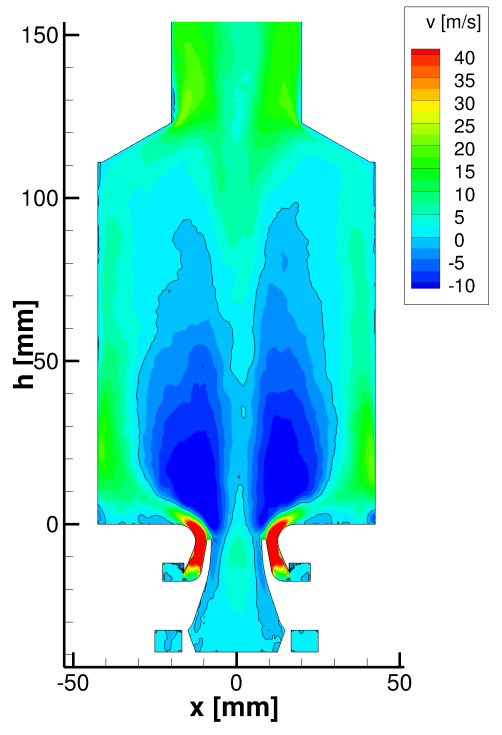

(e) $\dot{m}_{r} \rightarrow \infty$.

Figure 10: Mean axial velocity of the truncated burner simulations for different mass flow rate splits. Shown in these figures are the iso-lines for $v=0$ to indicate the region of separated and re-attached flow.

\section{Conclusions}

Large eddy simulations of a non-reacting flow in a gas turbine model combustor have been computed on different meshes. These meshes are categorized as pure hexahedral meshes and hybrid meshes consisting of hexahedral and tetrahedral elements. LES solutions on the pure hexahedral 


\begin{tabular}{cccc}
\hline & \multicolumn{3}{c}{ Swirl number } \\
\cline { 2 - 4 }$\dot{m}_{r}$ & Inner & Outer & Total \\
\hline \hline 0.0 & 0.420 & 0.000 & 0.407 \\
1.0 & 0.401 & 0.945 & 0.593 \\
1.4 & 0.397 & 0.903 & 0.686 \\
3.0 & 0.414 & 0.904 & 0.869 \\
$\infty$ & 0.000 & 0.951 & 0.961 \\
\hline
\end{tabular}

Table 3: Swirl numbers of the truncated burner simulations.

meshes show grid convergence and the predicted flow fields agree well with experimental measurements. Although similar agreement with experiments is also seen for the LES computation on the coarser hybrid mesh, the LES prediction on the finer hybrid mesh diverges. Pope's criterion indicates that the turbulence is sufficiently resolved on the finer mesh so the lack of mesh resolution may not be the cause for this behavior. Further examinations of the swirl numbers and the mass flow rate split between inner and outer swirler indicates that these parameters may not be fully responsible for the different simulation outcomes. In addition, LES calculations are also performed on a truncated geometry of the combustor. This is done to study the effects of the mass flow rate split on the flow field in the combustion chamber. The results of this investigation indicate that the increase in air flow through the outer swirler can lead to the attachment of the injector stream to the walls of the combustion chamber.

\section{Acknowledgment}

The authors gratefully acknowledge financial support through the NSF CAREER program with Award No. CBET-0844587 and the ONR under Grant No. N00014-10-1-0717. This work also used the Extreme Science and Engineering Discovery Environment (XSEDE), which is supported by National Science Foundation grant number OCI-1053575. The authors would like to thank Wolfgang Meier and Michael Stöhr for sharing the combustor-geometry and experimental data for model comparisons.

\section{References}

${ }^{1}$ Gupta, A. K., Lilley, D. G., and Syred, N., Swirl flows, Abacus Press, Jan. 1984.

${ }^{2}$ Vanoverberghe, K. P., Van Den Bulck, E. V., and Tummers, M. J., "Confined annular swirling jet combustion," Combustion Science and Technology, Vol. 175, No. 3, 2003, pp. 545-578.

${ }^{3}$ Vanierschot, M. and Van den Bulck, E., "Hysteresis in flow patterns in annular swirling jets," Experimental Thermal and Fluid Science, Vol. 31, No. 6, May 2007, pp. 513-524.

${ }^{4}$ Yee Chee See and Matthias Ihme, "Large-Eddy Simulation of a Gas Turbine Model Combustor," 51st AIAA Aerospace Sciences Meeting including the New Horizons Forum and Aerospace Exposition, Aerospace Sciences Meetings, American Institute of Aeronautics and Astronautics, Grapevine, Texas, Jan. 2013.

${ }^{5}$ Vreman, A. W., "An eddy-viscosity subgrid-scale model for turbulent shear flow: Algebraic theory and applications," Physics of Fluids, Vol. 16, No. 10, Sept. 2004, pp. 3670-3681.

${ }^{6}$ Ham, F., Mattsson, K., and Iaccarino, G., "Accurate and stable finite volume operators for unstructured flow solvers," Annual Research Briefs, Center for Turbulence Research, NASA Ames/Stanford University, 2006, pp. 243261.

${ }^{7}$ Ham, F., "An efficient scheme for large eddy simulation of low-Ma combustion in complex configurations," Annual Research Briefs (Center for Turbulence Research, Stanford University), 2007, pp. 41.

${ }^{8}$ Weigand, P., Meier, W., Duan, X., Stricker, W., and Aigner, M., "Investigations of swirl flames in a gas turbine model combustor: I. Flow field, structures, temperature, and species distributions," Combustion and Flame, Vol. 144, No. 1-2, Jan. 2006, pp. 205-224. 
${ }^{9}$ Meier, W., Duan, X., and Weigand, P., "Investigations of swirl flames in a gas turbine model combustor: II. Turbulence-chemistry interactions," Combustion and Flame, Vol. 144, No. 1-2, Jan. 2006, pp. 225-236.

${ }^{10}$ Widenhorn, A., Noll, B., and Aigner, M., "Numerical Study of a Non-Reacting Turbulent Flow in a Gas Turbine Model Combustor," 47 th AIAA Aerospace Sciences Meeting Including the New Horizons Forum and Aerospace Exposition, AIAA, Orlando, 2009.

${ }^{11}$ Beér, J. M. and Chigier, N. A., Combustion Aerodynamics, Krieger Publishing Company, 1972.

${ }^{12}$ Pope, S. B., "Ten questions concerning the large-eddy simulation of turbulent flows," New Journal of Physics, Vol. 6, March 2004, pp. 35-35.

${ }^{13}$ Yoshizawa, A., "Statistical theory for compressible turbulent shear flows, with the application to subgrid modeling," Physics of Fluids, Vol. 29, No. 7, July 1986, pp. 2152-2164.

${ }^{14}$ Boudier, G., Gicquel, L., Poinsot, T., Bissires, D., and Brat, C., "Comparison of LES, RANS and experiments in an aeronautical gas turbine combustion chamber," Proceedings of the Combustion Institute, Vol. 31, No. 2, Jan. 2007, pp. 3075-3082.

${ }^{15}$ Nicoud, F., Toda, H. B., Cabrit, O., Bose, S., and Lee, J., "Using singular values to build a subgrid-scale model for large eddy simulations," Physics of Fluids (1994-present), Vol. 23, No. 8, Aug. 2011, pp. 085106.

${ }^{16}$ Chigier, N. A. and Beér, J. M., "Velocity and Static-Pressure Distributions in Swirling Air Jets Issuing From Annular and Divergent Nozzles," Journal of Fluids Engineering, Vol. 86, No. 4, Dec. 1964, pp. 788-796.

${ }^{17}$ Billant, P., Chomaz, J.-M., and Huerre, P., "Experimental study of vortex breakdown in swirling jets," Journal of Fluid Mechanics, Vol. 376, 1998, pp. 183219. 